DISTRIBUTION STATEMENT A. Approved for public release; distribution is unlimited.

\title{
Cumulative and Synergistic Effects of Physical, Biological, and Acoustic Signals on Marine Mammal Habitat Use
}

\author{
Jennifer L. Miksis-Olds \\ Applied Research Laboratory \\ The Pennsylvania State University \\ PO Box 30 \\ State College, PA 16804 \\ phone: (814) 865-9318 fax: (814) 863-8783 email: jlm91@psu.edu \\ Jeffrey A. Nystuen \\ Applied Physics Laboratory \\ University of Washington \\ 1013 NE $40^{\text {th }}$ Street \\ Seattle, WA 98105 \\ phone:(206)-543-1343 email: nystuen@apl.washington.edu
}

Award Number: N000140810391

\section{LONG-TERM GOALS}

The long-term goal of this collaborative research effort is to enhance the understanding of how variability in physical, biological, and acoustic signals impact marine mammal habitat use. This is especially critical in areas like the Bering Sea where global climate change can lead to rapid changes of the entire ecosystem. Progressive climate change has the potential to expose areas of the Arctic that have been previously unavailable for civilian and military use. Baseline measurements will play an important role in mitigation efforts and environmental assessments as military activity increases in the region.

\section{OBJECTIVES}

The main objective of this work is to relate synoptic measurements of prey distribution, physical oceanographic process, and sound levels to marine mammal habitat use on the eastern Bering Sea shelf. Integrated data such as these will be vital in understanding the relationship between marine mammals and their environment both in the presence and absence of specific noise sources. Longterm measurements will play an important role in determining the point at which cumulative effects of the environment and human activities impact animal populations, and in identifying the conditions that pose the greatest risk.

The short-term objective in Year 2 of this project was to acquire and analyze acoustic data from two sub-surface NOAA moorings in the Bering Sea. This involved the detection and classification of marine mammal vocalizations, human activities, and geophysical signals from passive acoustic datasets. A time series of volume backscatter was also generated from different regions of the water column to provide insight on potential prey patterns corresponding to marine mammal presence and 


\section{Report Documentation Page}

Form Approved

OMB No. 0704-0188

Public reporting burden for the collection of information is estimated to average 1 hour per response, including the time for reviewing instructions, searching existing data sources, gathering and maintaining the data needed, and completing and reviewing the collection of information. Send comments regarding this burden estimate or any other aspect of this collection of information,

including suggestions for reducing this burden, to Washington Headquarters Services, Directorate for Information Operations and Reports, 1215 Jefferson Davis Highway, Suite 1204, Arlington

VA 22202-4302. Respondents should be aware that notwithstanding any other provision of law, no person shall be subject to a penalty for failing to comply with a collection of information if it

does not display a currently valid OMB control number.

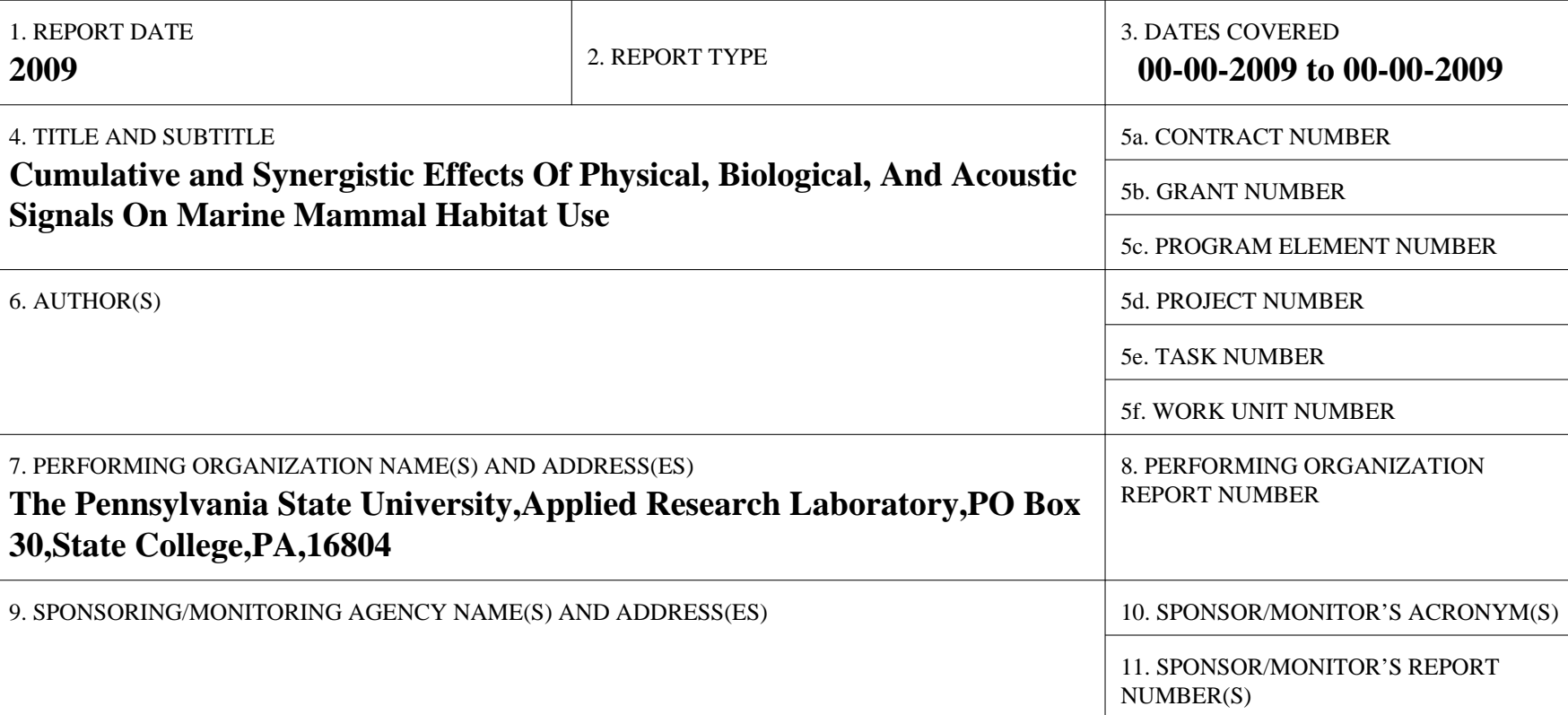

12. DISTRIBUTION/AVAILABILITY STATEMENT

Approved for public release; distribution unlimited

13. SUPPLEMENTARY NOTES

14. ABSTRACT

The long-term goal of this collaborative research effort is to enhance the understanding of how variability in physical, biological, and acoustic signals impact marine mammal habitat use. This is especially critical in areas like the Bering Sea where global climate change can lead to rapid changes of the entire ecosystem. Progressive climate change has the potential to expose areas of the Arctic that have been previously unavailable for civilian and military use. Baseline measurements will play an important role in mitigation efforts and environmental assessments as military activity increases in the region.

\section{SUBJECT TERMS}

16. SECURITY CLASSIFICATION OF:

a. REPORT unclassified b. ABSTRACT unclassified c. THIS PAGE unclassified
17. LIMITATION OF ABSTRACT

Same as Report (SAR)
18. NUMBER OF PAGES

9a. NAME OF

13 RESPONSIBLE PERSON 
vocal behavior. The objective of Year 3 will be to integrate the synoptic datasets in order to identify the combination of factors most strongly associated with marine mammal habitat use.

\section{APPROACH}

This project is a three-year study involving long-term monitoring of the physical and biological environment at two established NOAA mooring sites (known as M2 and M5) in the Bering Sea (Stabeno and Hunt, 2002). An acoustic monitoring system using both active and passive acoustic sensors was developed and deployed at the end of Year 1. The passive acoustic dataset is being used to assess the physical environment and to detect and identify marine mammal species and human activity present near the moorings. Passive recorders include Passive Aquatic Listeners (PALs) and PMEL recorders (AURALs). The PALs are adaptive sub-sampling instruments, where as the PMEL recorders produce a continuous time series. Comparison of the two datasets will determine how effective an adaptive sub-sampling technique is for detecting species diversity, what proportion of vocalizations is missed with the sub-sampling protocol, and whether appropriate parameters can be estimated to calculate the probability of detection for specific species. The active component is used to investigate zooplankton and fish distribution, patterns, and abundance. The active system includes a three frequency suite of scientific echosounders [Acoustic Water Column Profilers (AWCP)] that are deployed on each mooring $(125 \mathrm{kHz}, 200 \mathrm{kHz}$, and $460 \mathrm{kHz})$. Ancillary measurements of water column characteristics (current, temperature, salinity, nutrients, chlorophyll, etc.) will be available from the standard NOAA instrumentation on the moorings. Synoptic measurements from the multiple datasets will be integrated in Year 3 to provide insight into the factors influencing marine mammal habitat use.

Data analysis of ecosystem dynamics is a multi-step process. Patterns and interactions of measured variables will first be examined within each region according to the following sequence. 1) Datasets generated from the active and passive acoustic sensors will be analyzed separately to identify specific characteristics and patterns. 2) An analysis of covariance will be performed to quantify patterns of association between individual parameters. 3) A mixed-model analysis will be performed to identify relationships between marine mammal presence and environmental sound parameters, characteristics of physical processes, and prey abundance. Following the separate analyses in each of the two study regions, observed patterns and factors relating to habitat use will be compared between regions.

\section{WORK COMPLETED}

During the NOAA FOCI Mooring cruise in September 2008, a PAL was retrieved from mooring M5. This instrument returned a year of acoustic data spanning September 2007-September 2008.

Combination acoustic packages (each containing a PAL and three-frequency AWCP instrument suite) were then deployed on moorings M2 and M5 in water depths of $70 \mathrm{~m}$. A continuous AURAL recorder was also deployed on M5 by the National Marine Mammal Laboratory (Figure 1). The M2 instruments had an early deployment termination at the end of October 2008 due to a fishing trawler. The instruments were returned with no damage, but the AWCP cage had to be replaced. The M2 instruments were reconditioned and redeployed in April 2009. The instruments at M5 were retrieved, downloaded, reconditioned, and redeployed in May 2009. Mooring turnarounds and data retrieval at both sites is again planned for the last week in September 2009. 
Sampling parameters

The PALs deployed in September 2008 were programmed to sample and store averaged spectra every 9 minutes as a default. If signals of interest were detected (rain, drizzle, transient marine mammal vocalizations), a modified sampling protocol was triggered to increase the rate of sampling and record a 4.5 sec time series. Transient trigger criteria included a $+13 \mathrm{~dB}$ tonal peak between $800 \mathrm{~Hz}$ and 25 $\mathrm{kHz}$. Upon analysis of PAL data from the trawled instrument, the PAL sampling protocol was adjusted to trigger at a transient threshold level of $+12 \mathrm{~dB}$ and tonal peaks from $300 \mathrm{~Hz}-3 \mathrm{kHz}$ for upcoming deployments. Adjusting the transient trigger criteria allows for more focused sub-sampling of marine mammal vocalizations.

The sampling protocol for the AWCPs deployed in September 2008 consisted of a 5 minute sampling period every half hour. During the 5 minute sampling period, each frequency separately sampled for one minute totaling 3 minutes. The final two minutes of each sampling period was simultaneous sampling of all three frequencies. Active acoustic sampling by the AWCP sensors and ADCP was staggered to prevent acoustic interference of the two instrument sets. Analysis of the AWCP data from the trawled instruments indicated that there was no interference between the three frequencies during periods of simultaneous sampling. Subsequent deployment protocols were modified to eliminate individual frequency sampling and increase the time period of simultaneous sampling by all three frequency instruments.

Data Analysis

A project planning meeting focused on passive acoustic data analysis was held in Portland, OR (May 2009) and attended by all project participants. Categorization codes for sound sources were developed to maintain consistency between analysts, continuous and sub-sampled data, site locations, and years. An initial analysis strategy was developed for the PAL data consisting of a multilevel approach: 1) identification of sources triggering the alternative sampling protocol, 2) classification of incidental sources within the soundbite time series, 3) determination of daily species presence, 4) generation of seasonal soundscapes, and 5) generation of geophysical (wind, rain, etc) time series for the deployment period. Continuous data analysis is currently underway and will generate a dataset for comparison to the PAL data. Daily presence of species will be the first comparison parameter between the two datasets. The continuous recordings will also be analyzed to determine the proportion of time sound is generated during a calling bout of individual species. This information will then be used to estimate the probability of detection of the PAL sub-sampling algorithms.

Analysis of AWCP data differed slightly between the M2 and M5 sites due to prominent features of the water column. In October 2008, there was a pronounced and persistent pycnocline (Figure 2a). Acoustic backscatter was integrated over an hour for $2 \mathrm{~m}$ thick layers at the surface, in the upper water column, above the pycnocline, below the pycnocline, and near bottom. Data from M5 did not indicate the presence of a strong pycnocline, so acoustic backscatter was integrated over an hour for $2 \mathrm{~m}$ thick layers at $4 \mathrm{~m}, 15 \mathrm{~m}, 45 \mathrm{~m}$ and $55 \mathrm{~m}$ (Figure 2b).

\section{RESULTS}

$\underline{\text { Passive Acoustics }}$

Spectral data from M5 highlighted the change in seasonal soundscapes related to bowhead whale migration in January and the ice seal breeding season in March-May. The soundscapes of the summer and fall revealed an acoustic environment dominated by the sound of wind/waves compared to the winter and spring soundscapes that were dominated by marine mammals (Figure 3, Table 1). Regional 
differences in sound level and sources in the Bering Sea soundscapes is illustrated by comparing the October soundscapes from M5 and M2 in 2008 (Figure 4). Site M2 is louder and has greater variability. Additional results interpreting the geophysical signals from the spectral data can be found in the linked annual report by Jeffrey Nystuen, APL-UW.

Time series (or soundbites) recorded by the PAL provided information for identifying individual species. Soundbite allocation in the 2007-2008 dataset was limited to 16 soundbites per day unless previous days did not use the maximum number of soundbites. In this case, excess soundbites could be recorded on a single day until the reserve was depleted. Coverage of the limited soundbites was seasonal based on location and the number and type of species present (Figure 5, Table 1). Soundbites also tended to be triggered more often in periods of quite (Figure 6). Explanations for this include 1) the animals are exhibiting a natural noise compensation method by timing their vocal exchanges during period of quiet, and 2) signal detection is not as efficient during periods of elevated sound levels.

The temporal distribution of the most abundant marine mammals in the M5 region is shown in Figure 7. Analysis of the M5 2008-2009 data is in progress for comparison of temporal patterns across years at the same site. Table 1 shows the proportion of triggered soundbite classifications for a specific source (black) and the proportion of soundbites for specific sources detected incidentally in addition to the triggered source (red). Items of interest from Table 1 include these: 1 ) The soundscape at M5 in Oct. 2007 was mostly dominated by wind and by high levels of echolocation and humpback vocalizations. 2) There is a drastic change in the soundscape at M5 in April 2008 which is dominated by breeding ice seals. 3) At site M2 in Oct. 2008, there was a large number of right whale and shipping detections. All vocalizations detected from right whales were gunshots, not up calls. 4) Fin whales were detected incidentally 60\% of the time at M2 in Oct. 2008, but never triggered a soundbite recording. This was due to the fact that the minimum frequency for tonal triggers was $300 \mathrm{~Hz}$. In retrospect, this was desirable because fin whales have a high likelihood of being recorded incidentally when present due to their high vocalization rate, so that a lower frequency trigger threshold is not needed to detect this species. This reserves the limited soundbites for detecting species that are rare or have lower vocalization rates (e.g., right whales).

\section{Active Acoustics}

The variability of volume backscattering strength (Sv) was a function of location, layer depth, season, and ice cover. A strong vertical migration pattern was observed at M2 in Oct. 2008, while relatively less vertical migration was observed at M5 (Figure 8). The overall strength of vertical migration varied on seasonal time scales. Zooplankton abundance in deep water layers at M5 was more stable (had less variation) than surface and mid-water column layers (Figure 8). However, the zooplankton in the deep water layers had a much stronger response to a short-term disturbance in ice cover in Feb. 2009. For approximately 2 weeks in late Feb./early March, the ice at M5 retreated (Figure 9). The zooplankton response was less visible in the surface layer compared to the deep layers (Figure 10). The magnitude of the zooplankton bloom over the short time period of open water equaled or exceeded the average volume backscatter maximum values throughout the rest of the year. The onset of ice in early January corresponded to a zooplankton seasonal minimum in all layers (Figure 10).

Current analysis efforts include inverse modeling to estimate the size distribution of zooplankton within the identified layers. 


\section{IMPACT/APPLICATIONS}

The acoustic measurement system used in this project has the advantage of being deployed for long periods of time on subsurface moorings, affording the opportunity to collect valuable data during the harshest conditions of the winter season when traditional sampling techniques are not possible. The active acoustic dataset revealed that there is a rapid ecosystem response to relatively short-term changes in ice cover, which has a profound effect on zooplankton abundance in the deeper water column. How this and similar short-term environmental changes impact marine mammals is unknown at the present time. Identifying relationships between physical forcing mechanisms, biological activity, and marine mammal habitat use will not only be critical in understanding and ultimately predicting how marine mammals respond to noise, but also to how ecosystems respond to variability on multiple time scales.

The system used in this study is appropriate for use in almost all marine environments. It provides an advantage over continuous recording instruments in that the initial real-time processing of environmental sound by the PALs detects and identifies sources of interest without an overwhelming amount of data needing post-processing. The PALs and active acoustic sensors can be programmed to sample at the same time scale to ensure synoptic data collection. The adaptive sub-sampling protocol of the PAL is flexible and can incorporate a wide range of detection algorithms. A modified soundbite protocol will insure more representative coverage of time series throughout the day compared to the current sampling strategy that did not partition the soundbite acquisition.

\section{TRANSITIONS}

Underwater ambient sound contains quantifiable information about the marine environment, especially sea surface conditions including wind speed, rainfall rate and type, and sea state conditions (bubbles). Furthermore, the presence or absence of sea ice is has a distinctive acoustic signature in both the active and passive acoustic time series. This project represents a transition from the study of these sounds into the application of the physical and biological interpretation of the sound to provide quantitative assessment of the acoustic marine environment to address a higher level question: What is the effect of changes in the physical marine environment on marine mammal use of the habitat? This is a fundamental advance for practical use of passive acoustic monitoring of the underwater marine environment.

\section{RELATED PROJECTS}

\section{Monitoring sea surface processes using high frequency ambient sound, PI: Nystuen, APL UW ONR Award Number N00014-04-1-099}

The principal goal is to make passive acoustic monitoring of the marine environment an accepted quantitative tool for measuring sea surface conditions (wind speed, rainfall and sea state), monitoring for the presence and identity of marine wildlife (especially whales), and monitoring anthropogenic activities including shipping, sonar and other industrial activities. The new effort described here builds on the research of this ongoing project.

Several NOAA-supported projects, including Passive Acoustic monitoring of killer and beluga whales at the Barren Islands, Alaska, the Bering Sea Acoustic Report, Marine Mammal Monitoring for NW Fisheries, and Monitoring killer whale predation at Stellar Sea Lion rookeries in the Aleutian Islands, use PALs as the principal monitoring instrument for the description of the environment and for the detection 
an identification of marine cetaceans and other marine animals. This project benefits directly from the data collection strategies and interpretation developed for these projects.

\section{REFERENCES}

Stabeno, P.J. and G.L. Hunt, Jr. 2002: Overview of the Inner Front and Southeast Bering Sea carrying capacity programs. Deep-Sea Research: 49(26), 6157-6168

\section{PUBLICATIONS/PRESENTATIONS}

Miksis-Olds, JL. 2009. Passive acoustic monitoring of marine mammals using an adaptive, subsampling strategy. $3^{\text {rd }}$ Intergovernmental Conference: The Effects of Sounds in the Ocean on Marine Mammals. Lerici, Italy. Spetember 7-9.

Miksis-Olds, JL, Nystuen, JA, Parks, SE. 2009. Extracting incidental signals from targeted soundbites. $4^{\text {th }}$ International Workshop on Detection, Classification and Localization of Marine Mammals Using Passive Acoustics. Pavia, Italy. September 10-13.

Nystuen, JA, Hanson, MB, Emmons, C, Wade, P, Miksis-Olds, JL. 2009. Detection of killer whale presence usinf low duty cycle recorders. Journal of the Acoustical Society of America 125: 26162617.

Nystuen, JA, Miksis-Olds, JL, Parks, SE. 2009. Probability of the detection of calling bouts bby a low duty cycle passive acoustic recorder. $4^{\text {th }}$ International Workshop on Detection, Classification and Localization of Marine Mammals Using Passive Acoustics. Pavia, Italy. September 10-13.

Table 1. Classification of sources triggering soundbite recordings (black) and incidental detections (red) at mooring site M5 (Oct 2007 and Apr 2008) and M2 (Oct 2008). The $n$ values indicate the number of soundbites triggeres in each month.

\begin{tabular}{|c|c|c|c|c|c|c|c|c|}
\hline \multicolumn{3}{|c|}{ M5 Oct $2007(n=73)$} & \multicolumn{3}{|c|}{ M5 Apr $2008(n=240)$} & \multicolumn{3}{|c|}{ M2 Oct $2008(n=215)$} \\
\hline Echolocation & $28 \%$ & $5 \%$ & Bearded seal & $70 \%$ & $29 \%$ & Echolocation & $36 \%$ & $3 \%$ \\
\hline Wind & $20 \%$ & & Ribbon seal & $8 \%$ & $8 \%$ & Right whale & $26 \%$ & $1 \%$ \\
\hline Humpback & $20 \%$ & $20 \%$ & Bowhead & $5 \%$ & $5 \%$ & Ship & $14 \%$ & $12 \%$ \\
\hline Gray whale & $8 \%$ & $1 \%$ & Walrus & $5 \%$ & $19 \%$ & Mooring & $12 \%$ & $10 \%$ \\
\hline Mooring & $7 \%$ & $5 \%$ & Precipitation & $4 \%$ & $4 \%$ & Humpback & $3 \%$ & $4 \%$ \\
\hline Precipitation & $7 \%$ & $7 \%$ & Low clicks & $2 \%$ & $20 \%$ & Killer whale & $3 \%$ & $1 \%$ \\
\hline & & & Echolocation & & $20 \%$ & Precipitation & $2 \%$ & $8 \%$ \\
\hline & & & & & & Fin whale & & $60 \%$ \\
\hline
\end{tabular}




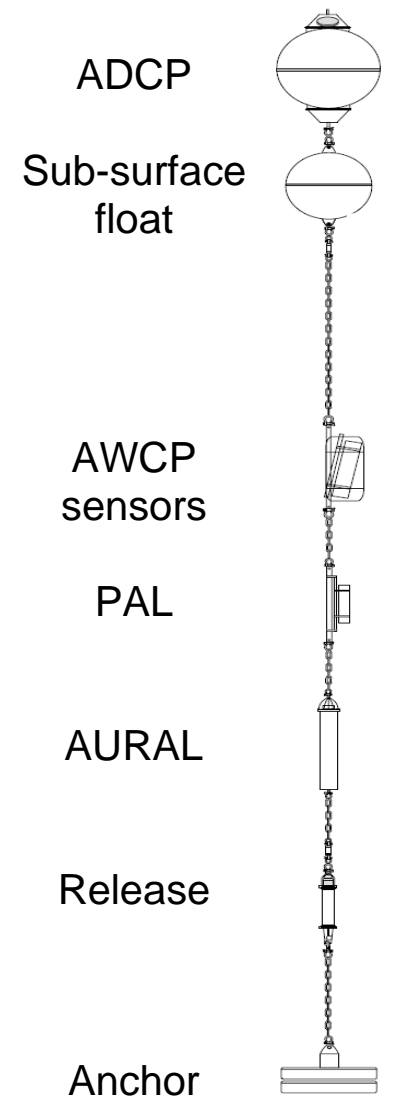

Figure 1. Sub-surface mooring configuration at site M5.
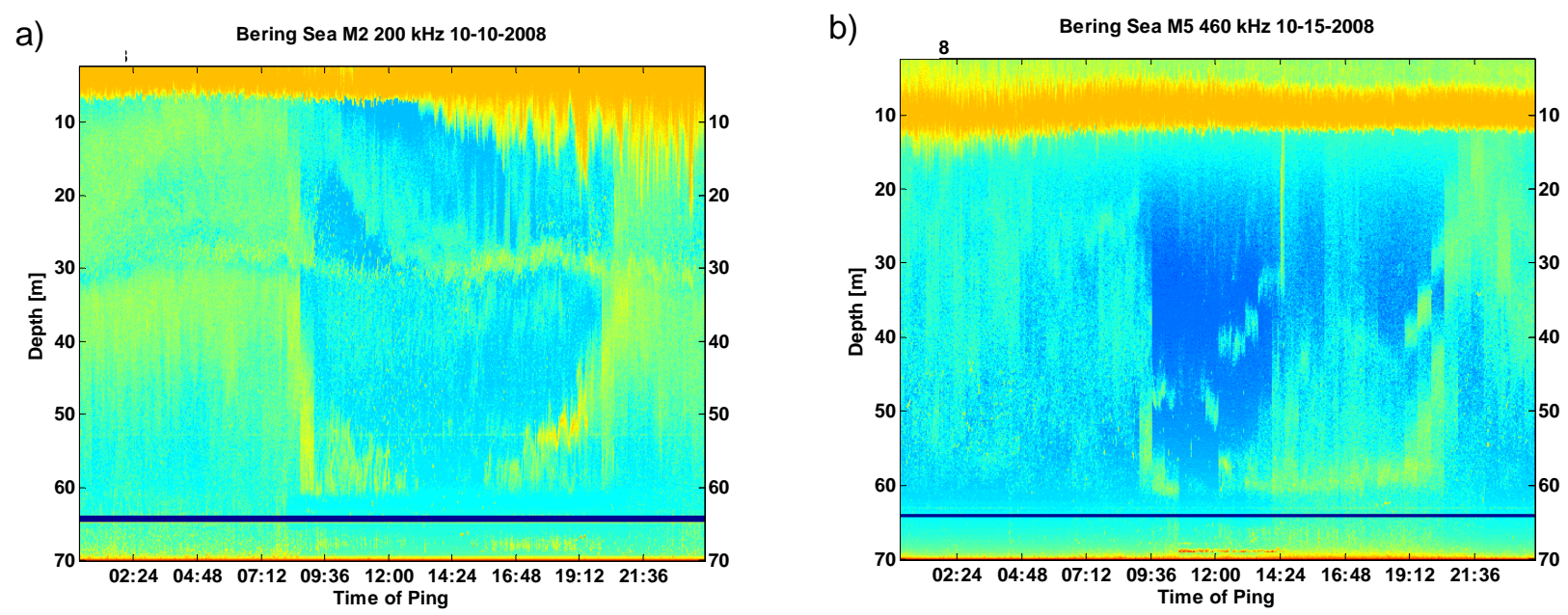

Figure 2. a) representative 24 hour echogram from M2 on 10/10/2008 at $200 \mathrm{kHz}$. Note visible zooplankton layer concentrated at the pycnocline. b) representative 24 hour echogram from M5 on 10/15/2008 at $460 \mathrm{kHz}$. 
a)

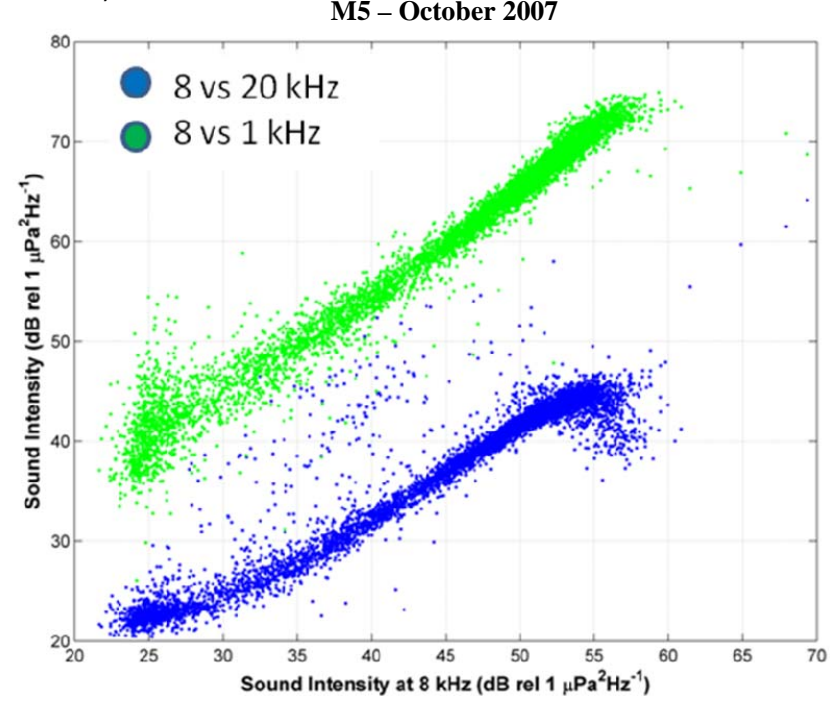

c)

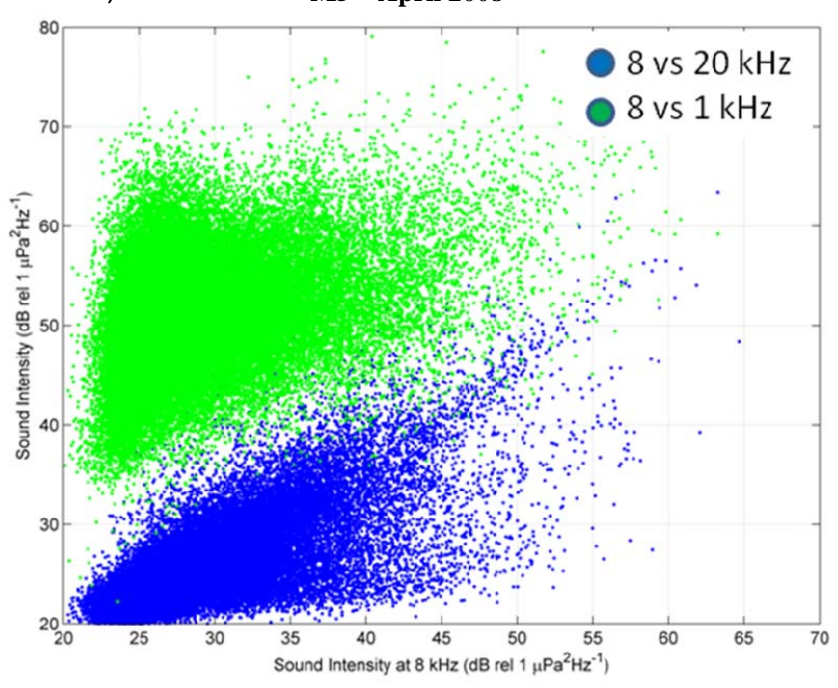

b)

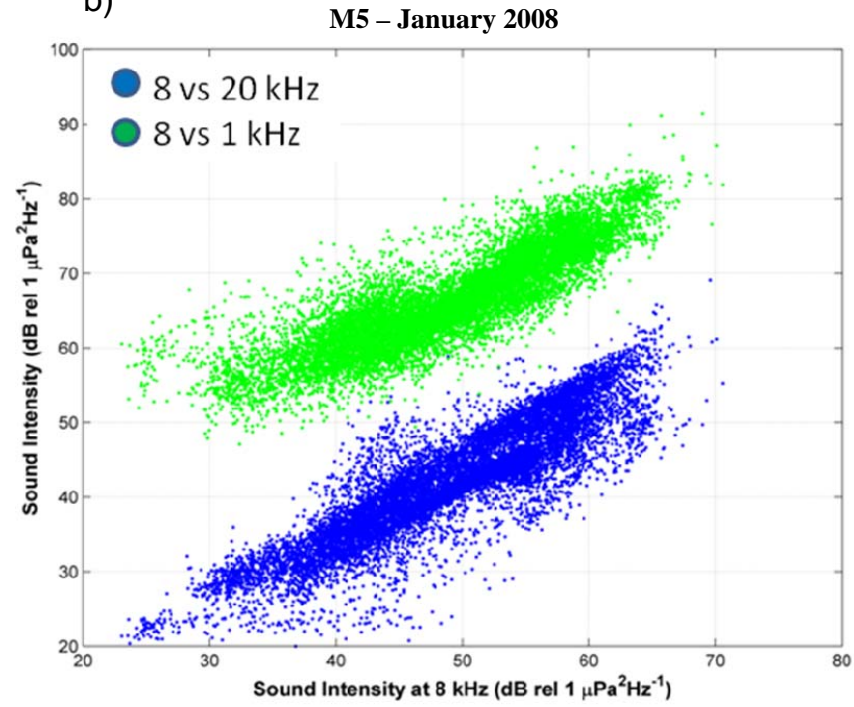

d)

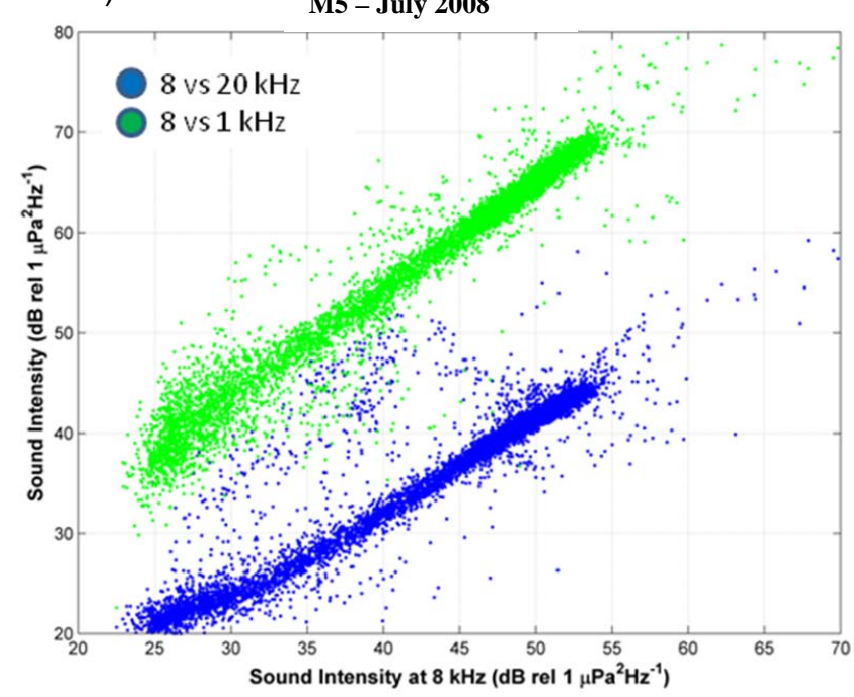

Figure 3. Seasonal soundscapes generated from spectral data. The $x$ axis in all panels is the sound level at $8 \mathrm{kHz}$. The y axis is the sound level at either $20 \mathrm{kHz}$ (blue) or $1 \mathrm{kHz}$ (green). The soundscapes in fall (a) and summer (d) show a linear pattern indicating an environment dominated by wind. Sound levels increase linearly as wind speed increases. The spectral variability in winter (b) is due to bowhead song, while in spring (c) vocalizations from breeding ice seals dominate the soundscape. 
a)

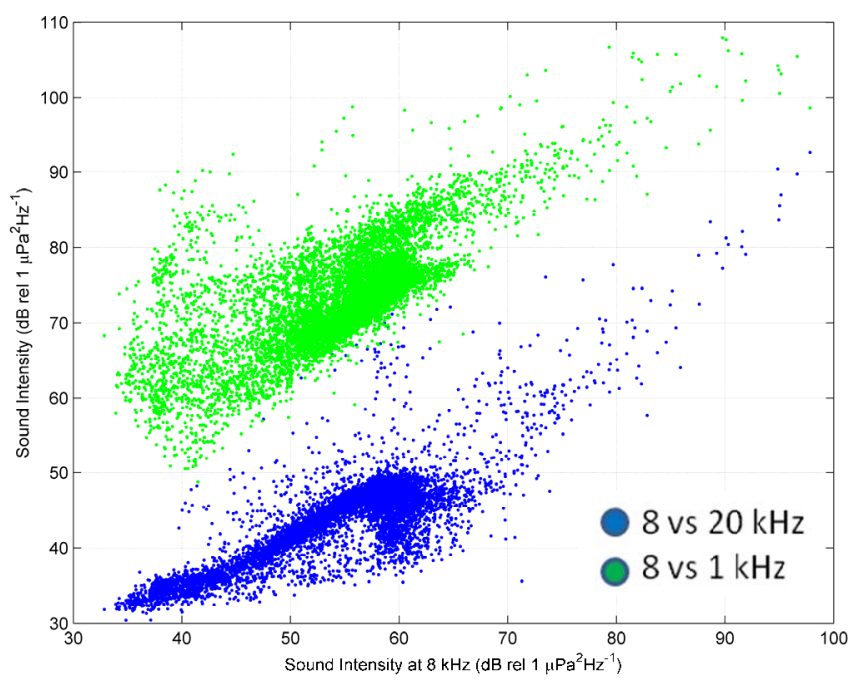

b)

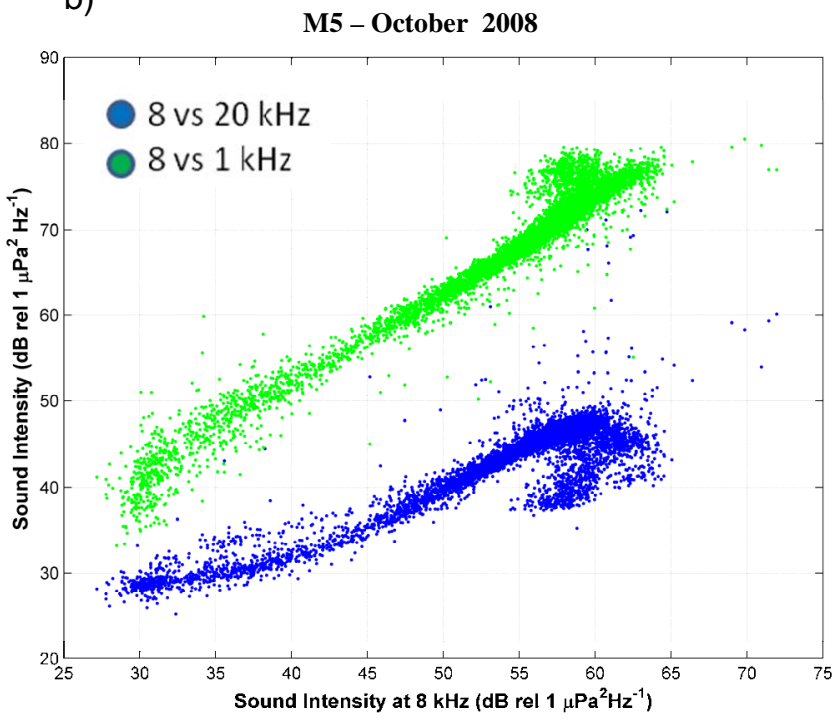

Figure 4. October 2008 soundscapes from site M2 (a) and M5 (b). The x axes are the sound level at $8 \mathrm{kHz}$. The y axes are the sound level at either $20 \mathrm{kHz}$ (blue) or $1 \mathrm{kHz}$ (green). The soundscape at M5 show a linear pattern indicating an environment dominated by wind. Sound levels increase linearly as wind speed increases. The variabiliy at M2 is due to the presnece of vocalizing marine mammals and ships (Table 1).

\section{M5 2007-2008}

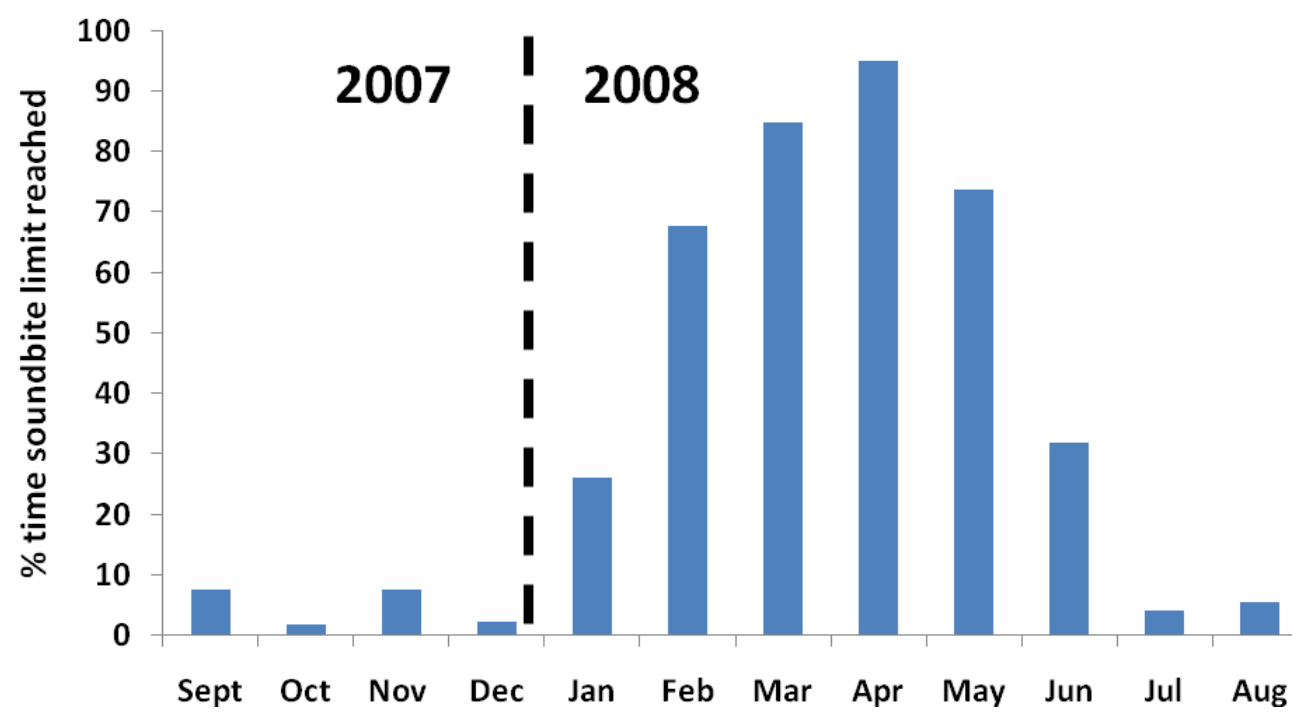

Figure 5. Distribution of monthly soundbite coverage for a year deployment at site m5. 

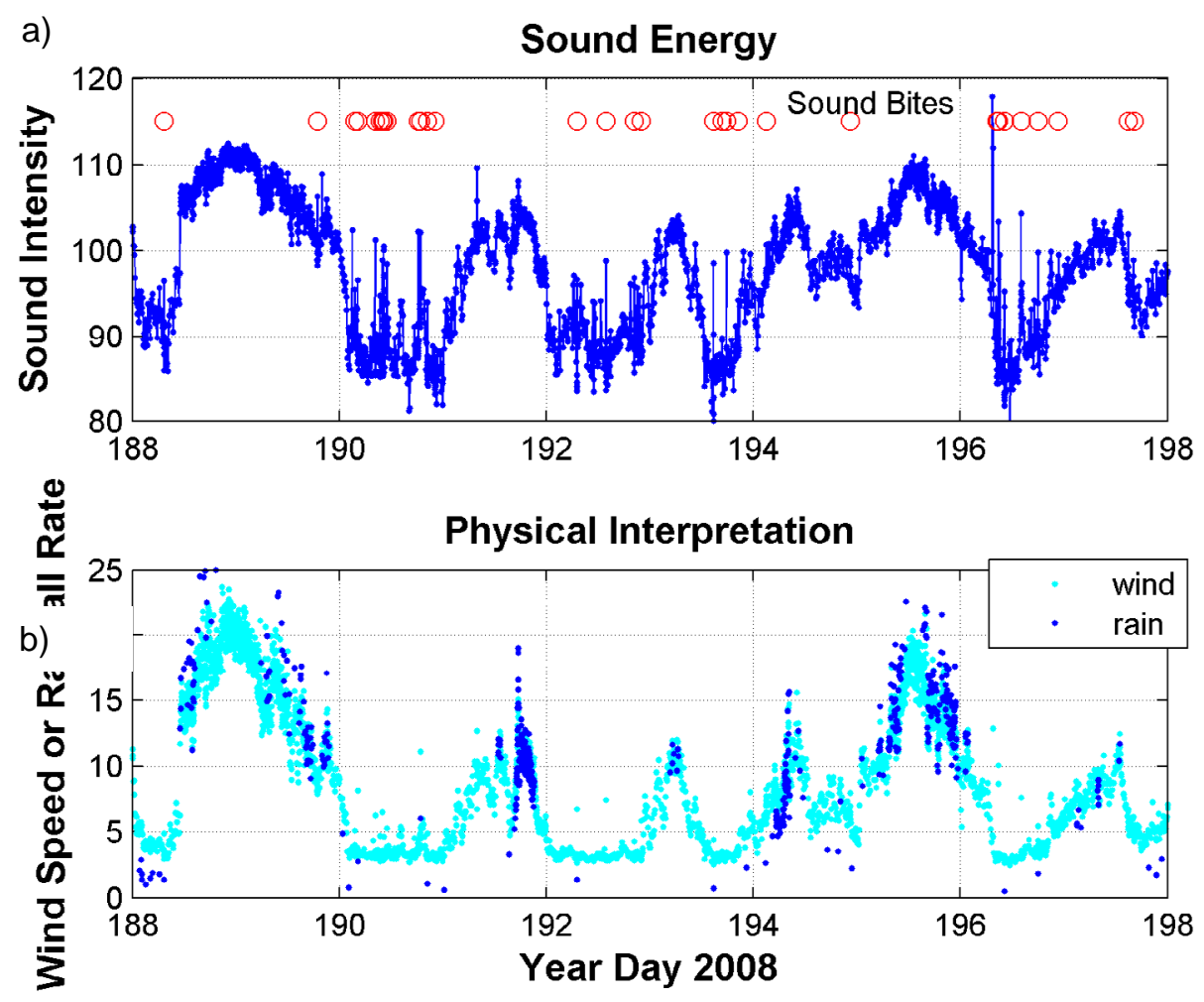

Figure 6. Ten days of spectral data from July 2008.. (a) Sound intensity (dB re $1 \mu \mathrm{Pa}$ ) and time of sound bite triggers (red circles) over the 10 day period. (b) Physical interpretation of of spectral data over the same time period. Wind speed $(\mathrm{m} / \mathrm{s})$. Rainfall rate $(\mathrm{mm} / \mathrm{hr})$.

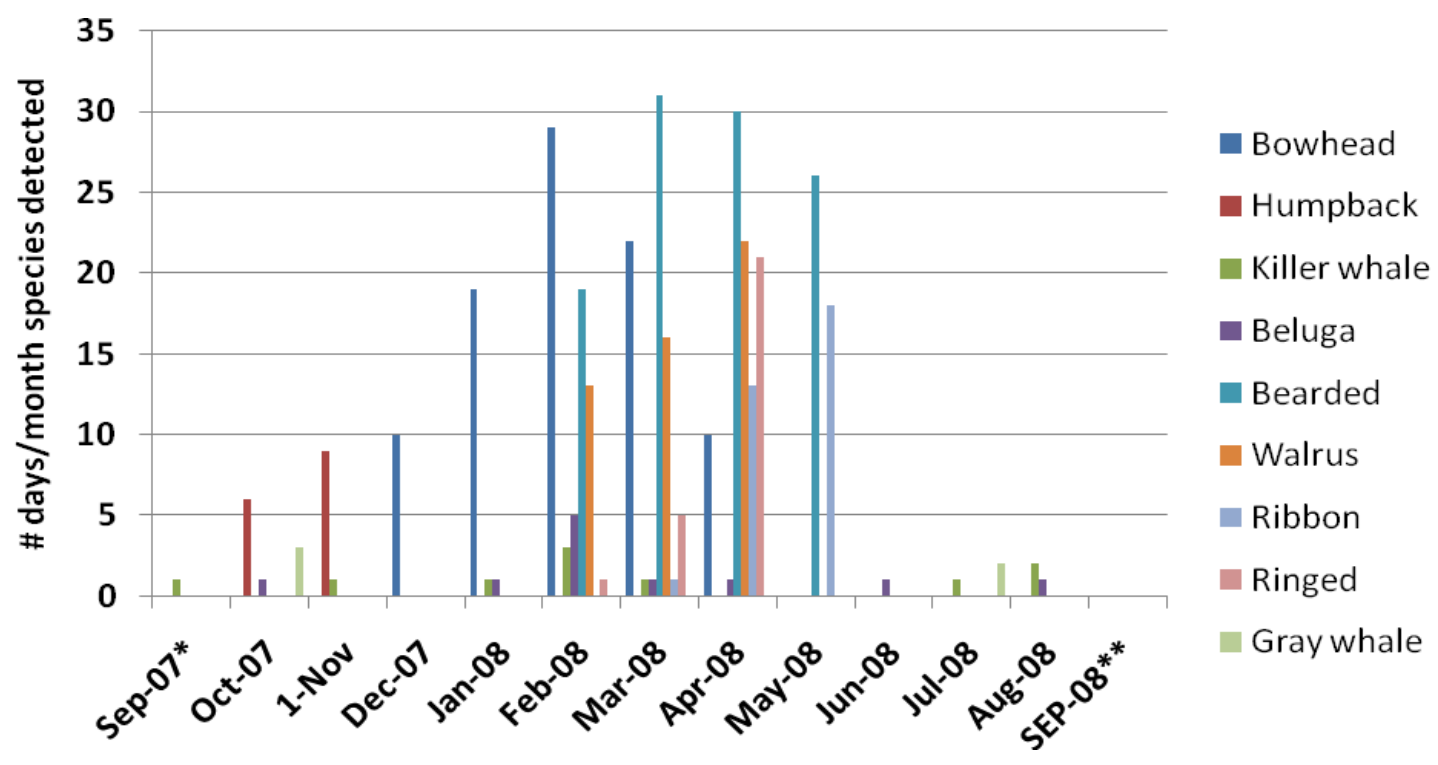

Figure 7. Species distribution determined from detections in soundbites at M5 from 2007-2008. * indicates that only 10 days were sampled in this month. ** indacates that only 2 days were sampled. 
a)
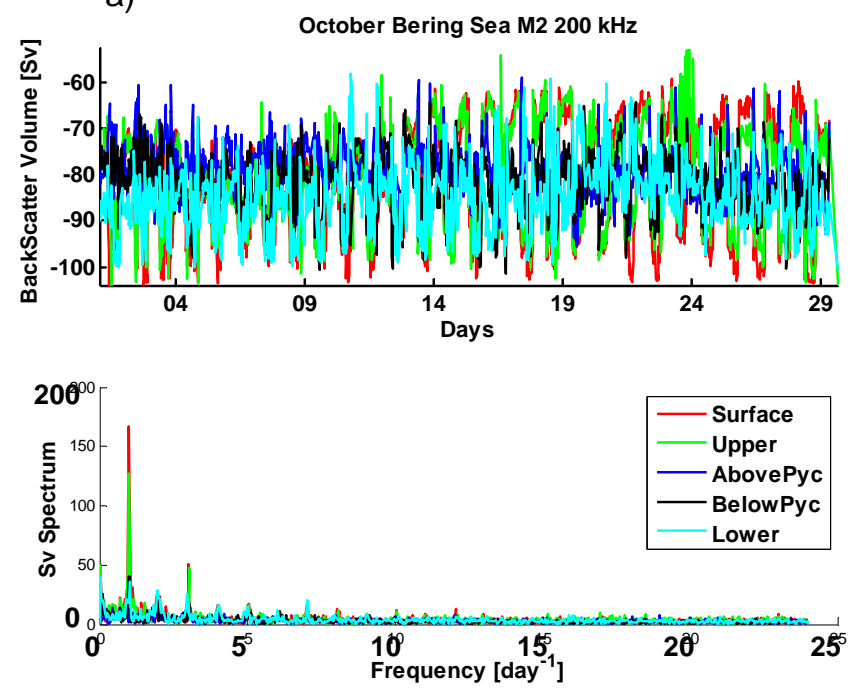

b)
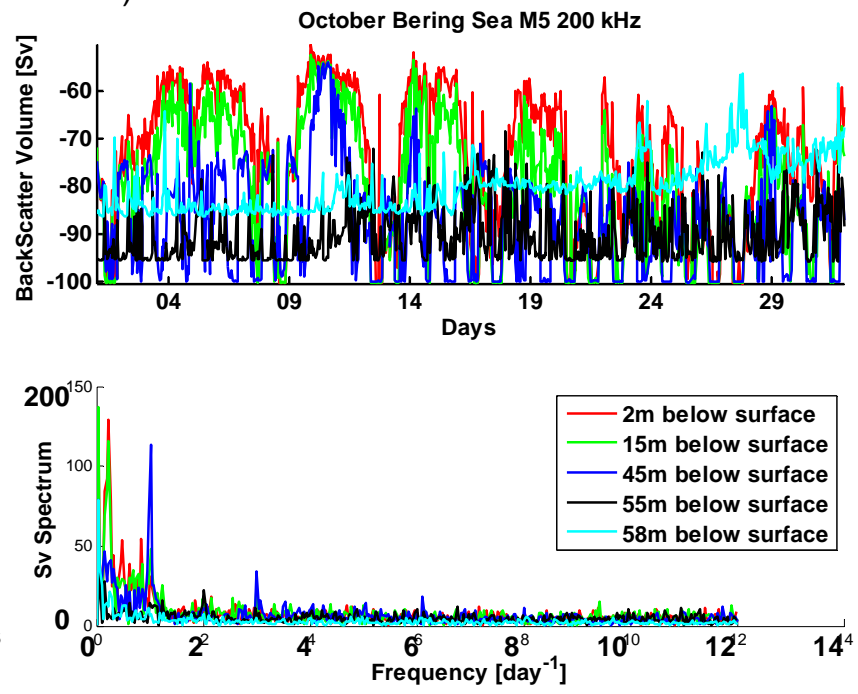

Figure 8. $200 \mathrm{kHz}$ Sv and spectral pattern from M2 (a) and M5 (b) in October 2008. M2 shows a strong daily pattern for all depth layers. The temporal pattern at M5 shows a strong daily cycle for layers at depths of $45 \mathrm{~m}$ and $55 \mathrm{~m}$. The temporal pattern at other layers is not as clear. 
a)

\section{Percent Ice Cover}

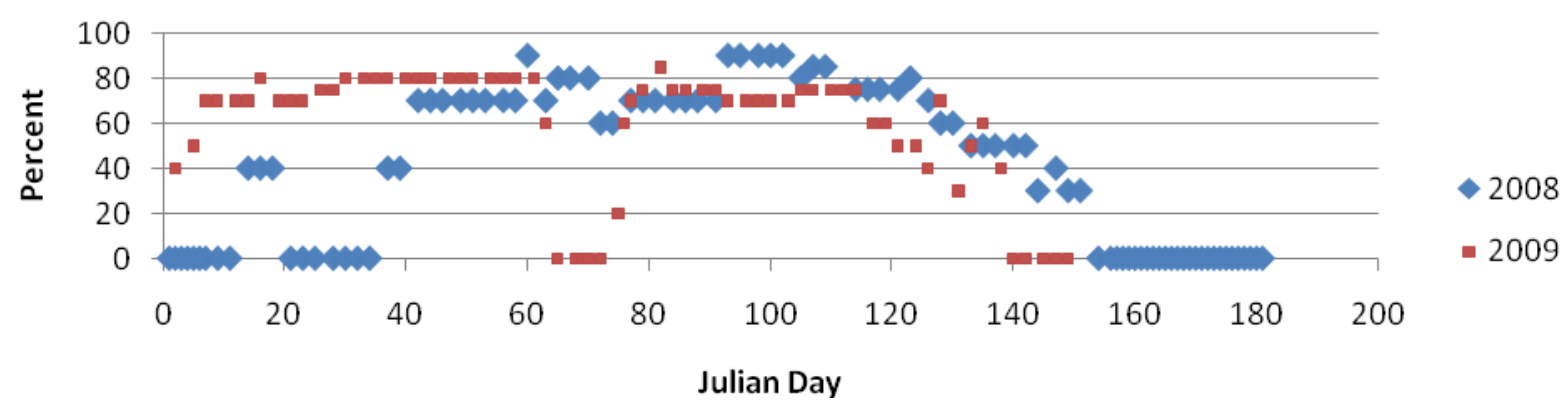

b)

Average Ice Thickness

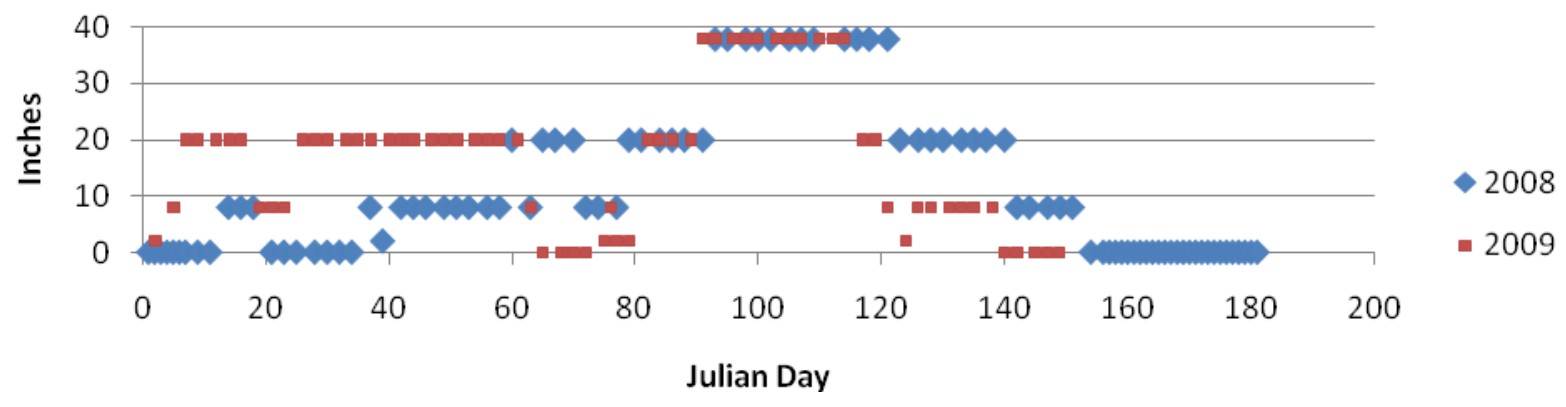

Figure 9. Percent ice cover (a) and average ice thickness (b) at site M5 in 2008 and 2009. Ice dat was obtained from the Ice Desk of the National Weather Service (US Dept of Commerce, NOAA). Note 2 weeks of ice retreat in late February/early March in 2009. 
a)

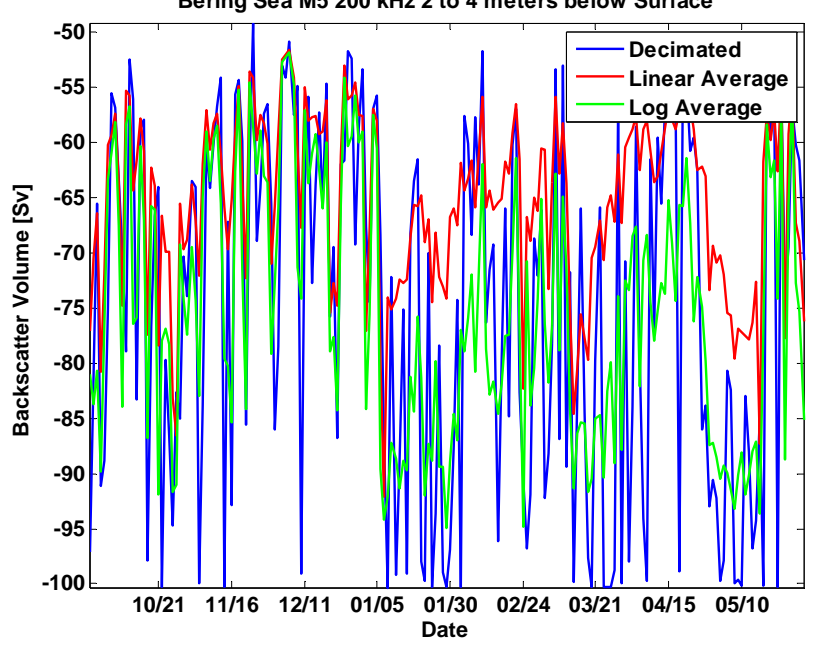

c)

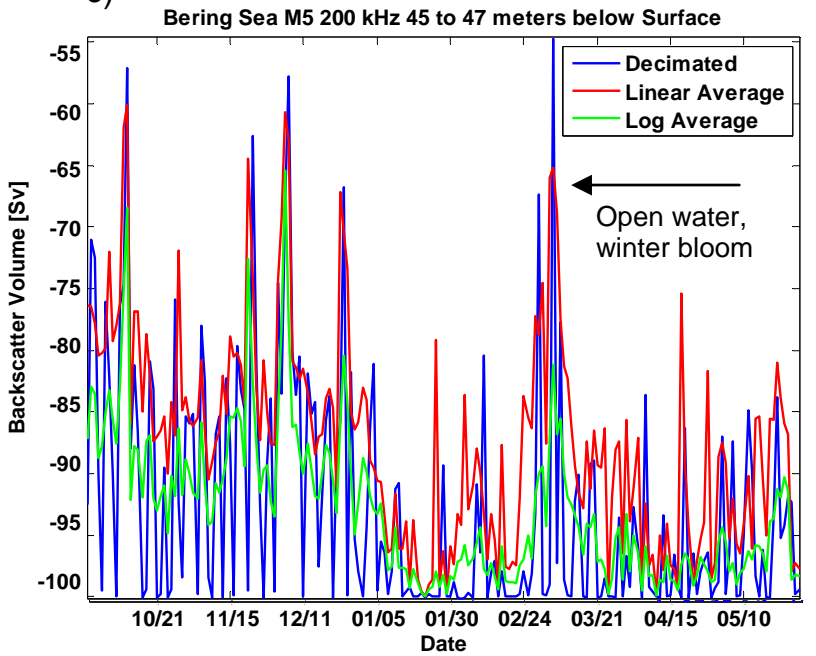

b)

Bering Sea M5 $200 \mathrm{kHz} 15$ to 17 meters below Surface

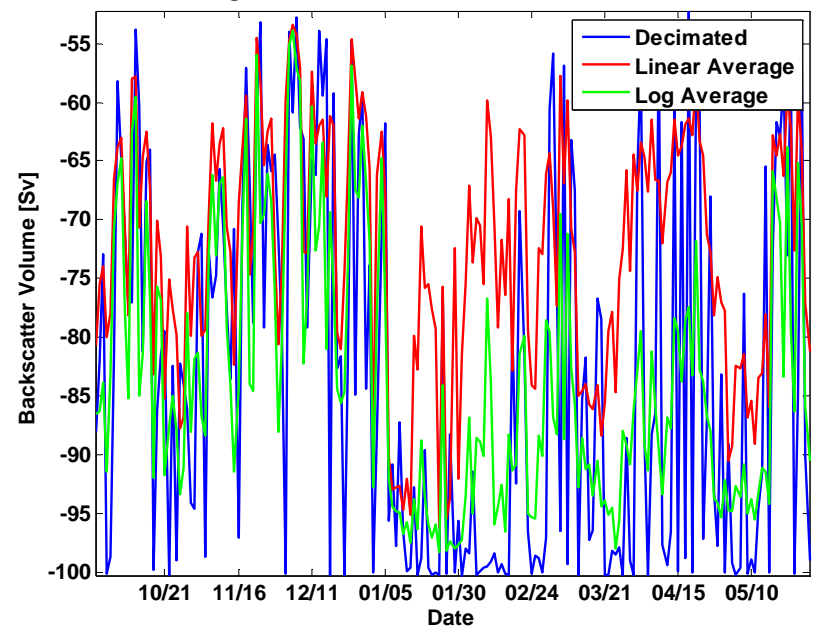

d)

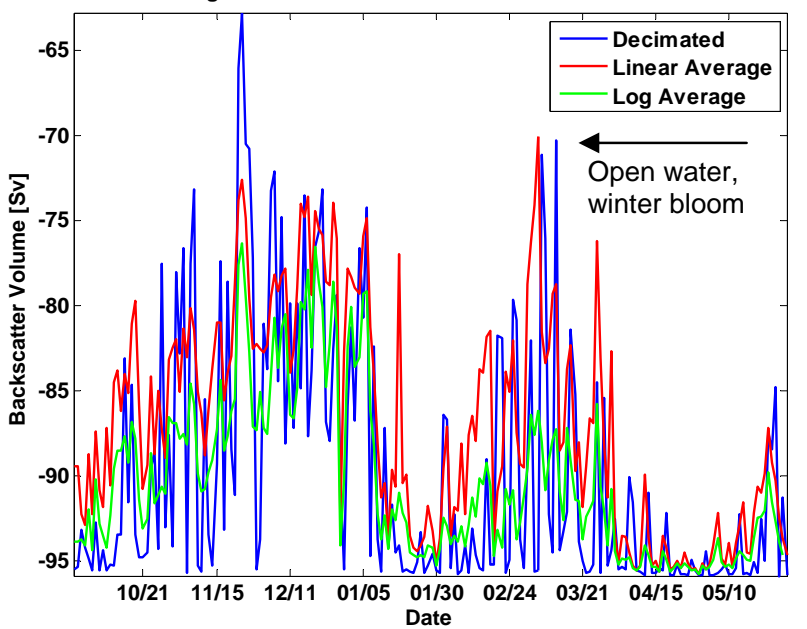

Figure 10. Nine month $200 \mathrm{kHz} S v$ time series at M5 from 9/08 through 5/09. (a) surface layer. (b) $15 \mathrm{~m}$ below surfacce. (c) $45 \mathrm{~m}$ below surface. (d) bottom layer. Blue lines refelct a 24 hour decimation. Red lines present a 24 hour linear average. Green lines refelct a 24 hour log average. 